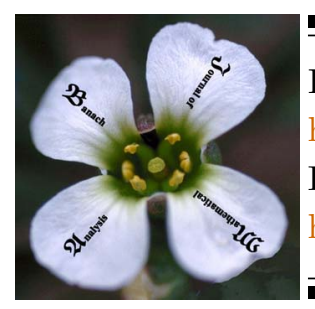

Banach J. Math. Anal. 10 (2016), no. 1, 133-146

http://dx.doi.org/10.1215/17358787-3345005

ISSN: $1735-8787$ (electronic)

http://projecteuclid.org/bjma

\title{
ON THE SUBMULTIPLICATIVITY AND SUBADDITIVITY OF THE SPECTRAL AND ESSENTIAL SPECTRAL RADIUS
}

\author{
MARKO KANDIĆ $^{1 *}$ and ALJOŠA PEPERKO ${ }^{2}$ \\ Communicated by M. Neumann
}

\begin{abstract}
Suppose that $A$ and $B$ are positive operators on an ordered Banach space with a normal and generating cone satisfying $0 \leq A B \leq B A$. It is known that, in this case, we have

$$
r(A B) \leq r(A) r(B) \quad \text { and } \quad r(A+B) \leq r(A)+r(B) .
$$

In this article we consider less restrictive assumptions on $A$ and $B$ which give us the same inequalities as above. Moreover, we also prove these inequalities in a more general setting of ordered algebras, and we consider related results in the Banach algebra setting. We apply our results to the essential spectral radius $r_{\text {ess }}$ of AM-compact operators and prove the equality $r_{\text {ess }}(A B+B A)=2 r_{\text {ess }}(A B)$ under reasonable assumptions.
\end{abstract}

\section{IntRoduction}

It is well known that the inequalities

$$
r(A B) \leq r(A) r(B) \quad \text { and } \quad r(A+B) \leq r(A)+r(B)
$$

(for the spectral radius $r$ ) hold for arbitrary commuting bounded operators $A$ and $B$ on every Banach space $X$. It is also known that inequalities in (1.1) hold if $A$ and $B$ are positive operators on an ordered Banach space with a normal and

Copyright 2016 by the Tusi Mathematical Research Group.

Received Dec. 15, 2014; Accepted Apr. 23, 2015.

${ }^{*}$ Corresponding author.

2010 Mathematics Subject Classification. Primary 47A10; Secondary 47B47, 47B60, 47B65.

Keywords. AM-compact operators, spectral radius inequalities, ordered algebras, essential spectral radius, commutator. 
generating cone satisfying $0 \leq A B \leq B A$ (see, e.g., [22] or [23]). In this article, we find other less restrictive assumptions under which these inequalities hold. Closely related conditions to our assumptions have already appeared in different contexts (see, e.g., [13], [12], [15], [8], and the references therein).

The paper is organized as follows. In Section 3 we prove (1.1) in a general setting of ordered algebras, and in Section 4 we consider related results in the setting of Banach algebras. In Sections 5 and 6 we apply our results to the spectral radius of positive operators and to the essential spectral radius $r_{\text {ess }}$ of AM-compact operators. Moreover, we also prove that the equality $r_{\mathrm{ess}}(A B+B A)=2 r_{\mathrm{ess}}(A B)$ holds under reasonable assumptions (Theorems 6.8 and 6.9).

\section{PRELiminaries}

Let $E$ be an ordered vector space. By $E^{+}$we denote the set of all positive vectors in $E$. If $p$ is a seminorm on $E$, then the positive cone $E^{+}$is called $p$-normal whenever there exists $\gamma>0$ such that $0 \leq x \leq y$ implies $p(x) \leq \gamma p(y)$. If $p$ is a norm on an ordered vector space $E$, we will refer to a $p$-normal cone simply as a normal cone.

An ordered vector space $\mathcal{A}$ that is an algebra as well, is said to be an ordered algebra whenever $\mathcal{A}^{+} \cdot \mathcal{A}^{+} \subseteq \mathcal{A}^{+}$and $1 \in \mathcal{A}^{+}$whenever $\mathcal{A}$ is unital. If $\mathcal{A}$ is a nonunital ordered algebra, we can extend the ordering to the standard unitization $\mathcal{A}_{1}$ by $(a, \lambda) \geq(0,0)$ whenever $a \in \mathcal{A}^{+}$and $\lambda \geq 0$. If $a$ is an element in a nonunital algebra $\mathcal{A}$, then we denote by $a_{1} \in \mathcal{A}_{1}$ the element $(a, 0) \in \mathcal{A}_{1}$. If $p$ is a seminorm on a nonunital algebra $\mathcal{A}$, then we denote by $p_{1}$ the seminorm on $\mathcal{A}_{1}$ defined by $p_{1}((a, \lambda))=p(a)+|\lambda|$. If $p$ is submultiplicative on $\mathcal{A}$, then it should be clear that $p_{1}$ is submultiplicative on $\mathcal{A}_{1}$.

Let $p$ be a seminorm on a partially ordered algebra $\mathcal{A}$. It is not hard to see that whenever $\mathcal{A}$ is a nonunital ordered algebra with a $p$-normal positive cone, then the positive cone $\mathcal{A}_{1}^{+}$of the unitization $\mathcal{A}_{1}$ is $p_{1}$-normal.

Let $\mathcal{A}$ be an algebra, and let $p$ be a submultiplicative seminorm on $\mathcal{A}$. The p-spectral radius $r_{p}$ on $\mathcal{A}$, defined by

$$
r_{p}(a)=\limsup _{n \rightarrow \infty} p\left(a^{n}\right)^{\frac{1}{n}}
$$

satisfies $r_{p}(a) \leq p(a)$ and is equal to

$$
r_{p}(a)=\lim _{n \rightarrow \infty} p\left(a^{n}\right)^{\frac{1}{n}}=\inf _{n \in \mathbb{N}} p\left(a^{n}\right)^{\frac{1}{n}} .
$$

Indeed, if we denote $p_{n}=p\left(a^{n}\right)$, then submultiplicativity of $p$ implies that we have

$$
p_{n+m} \leq p_{n} p_{m}
$$

for all $n, m \in \mathbb{N}$. A well-known calculus lemma asserts that, for any sequence of nonnegative real numbers $\left\{p_{n}\right\}_{n \in \mathbb{N}}$ which satisfy inequalities in (2.1) for all $n, m \in \mathbb{N}$, we have

$$
\lim _{n \rightarrow \infty} p_{n}^{\frac{1}{n}}=\inf _{n \in \mathbb{N}} p_{n}^{\frac{1}{n}} .
$$


It is not hard to see that, for an arbitrary element $a$ in a nonunital algebra $\mathcal{A}$ with a submultiplicative seminorm $p$, we have $r_{p_{1}}\left(a_{1}\right)=r_{p}(a)$. The element $a \in \mathcal{A}$ is called $p$-quasinilpotent if $r_{p}(a)=0$ and it is called $p$-power bounded if there exists a positive constant $N>0$ such that $p\left(a^{n}\right) \leq N$ for every $n \in \mathbb{N}$. If $a$ is $p$-power bounded, then $r_{p}(a) \leq 1$.

The equality $(b a)^{n}=b(a b)^{n-1} a$ implies $r_{p}(a b)=r_{p}(b a)$ for all $a, b \in \mathcal{A}$. Throughout the article we use the notation $a^{0} b=b=b a^{0}$.

For other relevant facts and definitions on ordered vector spaces, ordered algebras, ordered Banach spaces, and Banach lattices and operators acting on them, we refer the reader to [3], [4], and [18].

\section{3. $p$-SPECTRAL RADIUS INEQUALITIES}

The proof of the following result on the monotonicity of the $p$-spectral radius is straightforward (and similar to the proof of [18, Theorem 4.1]) and is therefore omitted.

Lemma 3.1. Let $\mathcal{A}$ be an ordered algebra, and let $\mathcal{B}$ be its subalgebra. Let $p$ be a submultiplicative seminorm on $\mathcal{B}$ such that the subcone $\mathcal{B}^{+}=\mathcal{B} \cap \mathcal{A}^{+}$is p-normal. If $a, b \in \mathcal{A}^{+}$and $n_{0} \in \mathbb{N}$ are such that $a^{n}, b^{n} \in \mathcal{B}^{+}$and $a^{n} \leq b^{n}$ for all $n \geq n_{0}$, then $r_{p}(a) \leq r_{p}(b)$.

The following two theorems generalize well-known results on the submultiplicativity and subadditivity of the spectral radius in several directions.

Theorem 3.2. Let $\mathcal{A}$ be an ordered algebra, let $p$ be a submultiplicative seminorm on $\mathcal{A}$, and let $k \in \mathbb{N}_{0}$. Let $\mathcal{B}$ be a subalgebra in $\mathcal{A}$ ordered by a p-normal cone $\mathcal{B}^{+}=\mathcal{A}^{+} \cap \mathcal{B}$ which satisfies $\mathcal{A}^{+} \mathcal{B}^{+} \mathcal{A}^{+} \subseteq \mathcal{B}^{+}$. Let $a, b \in \mathcal{A}^{+}$, and suppose that there exists a p-power bounded element $c \in \mathcal{A}^{+}$such that $a c \leq c a, b c \leq c b$, and $a b \leq c b a$. If at least one of the elements $a$ and $b$ is contained in $\mathcal{B}^{+}$, then

$$
\begin{aligned}
r_{p}\left(c^{k} b a\right) & \leq r_{p}(a) r_{p}(b), \quad r_{p}(a b) \leq r_{p}(a) r_{p}(b) \quad \text { and } \\
r_{p}(c b a-a b) & \leq r_{p}(c b a) \leq r_{p}(a) r_{p}(b) .
\end{aligned}
$$

If, in addition, $p(c)<1$, then elements $a b, b a, c^{k} b a$, and $c b a-a b$ are $p$-quasinilpotent.

Proof. At first, we will prove by induction on $n$ that the inequality

$$
\left(c^{k} b a\right)^{n} \leq c^{\frac{n(n-1+2 k)}{2}} b^{n} a^{n}
$$

holds for all $n \in \mathbb{N}$ and $k \in \mathbb{N}_{0}$. For $n=1$, inequality (3.3) is obvious. Assume now that inequality (3.3) holds for some positive integer $n$. Then we have

$$
\left(c^{k} b a\right)^{n+1} \leq c^{\frac{n(n-1+2 k)}{2}} b^{n} a^{n} c^{k} b a \leq c^{\frac{n(n-1+2 k)}{2}+k+n} b^{n+1} a^{n+1}=c^{\frac{(n+1)(n+2 k)}{2}} b^{n+1} a^{n+1},
$$

and the induction step is completed.

Since the cone $\mathcal{B}^{+}$is $p$-normal, there exists a positive constant $\gamma>0$ such that $p(x) \leq \gamma p(y)$ whenever $0 \leq x \leq y$ holds in $\mathcal{B}$. The $p$-power boundedness of the element $c$ implies that there exists a positive constant $N>0$ such that $p\left(c^{n}\right) \leq N$ 
for every $n \in \mathbb{N}$. Since $\mathcal{B}^{+}$satisfies $\mathcal{A}^{+} \mathcal{B}^{+} \mathcal{A}^{+} \subseteq \mathcal{B}^{+}$, it follows that both sides of inequality (3.3) are contained in $\mathcal{B}^{+}$and so

$$
p\left(\left(c^{k} b a\right)^{n}\right) \leq \gamma p\left(c^{\frac{n(n-1+2 k)}{2}} b^{n} a^{n}\right)
$$

for all $n \in \mathbb{N}, n \geq 2$. Since $p$ is submultiplicative and $c$ is $p$-power bounded, we have

$$
p\left(\left(c^{k} b a\right)^{n}\right) \leq \gamma p\left(c^{\frac{n(n-1+2 k)}{2}}\right) p\left(b^{n}\right) p\left(a^{n}\right) \leq \gamma N p\left(b^{n}\right) p\left(a^{n}\right)
$$

for all $n \in \mathbb{N}, n \geq 2$. By taking the $n$th roots in inequality (3.4) and letting $n$ go to infinity, we obtain $r_{p}\left(c^{k} b a\right) \leq r_{p}(b) r_{p}(a)$. When $k=0$ we obtain also the second inequality in (3.1) since $r_{p}(b a)=r_{p}(a b)$, and when $k=1$ we obtain the right-hand side of inequality (3.2). Inequality (3.2) follows from the fact that $0 \leq$ $c b a-a b \leq c b a$ and $0 \leq(c b a-a b)^{n} \leq(c b a)^{n}$ for all $n \in \mathbb{N}$. Since $(c b a-a b)^{n} \in \mathcal{B}^{+}$ and $(c b a)^{n} \in \mathcal{B}^{+}$for all $n \geq 3$, the $p$-normality of the cone $\mathcal{B}^{+}$implies (3.2) by Lemma 3.1.

Suppose now that $p(c)<1$ and $k \in \mathbb{N}_{0}$. From inequality (3.4) we obtain

$$
p\left(\left(c^{k} b a\right)^{n}\right)^{\frac{1}{n}} \leq \gamma^{\frac{1}{n}} p(c)^{\frac{n-1+2 k}{2}} p\left(b^{n}\right)^{\frac{1}{n}} p\left(a^{n}\right)^{\frac{1}{n}} .
$$

Since $p(c)<1$, the right-hand side of inequality (3.5) tends to zero when $n \rightarrow$ $\infty$, so that we obtain $r_{p}\left(c^{k} b a\right)=0$. When $k=0$ we obtain that $a b$ and $b a$ are $p$-quasinilpotent, and when $k=1$ we obtain that $c b a$ is $p$-quasinilpotent. Inequality (3.2) implies $r_{p}(c b a-b a)=0$, which completes the proof.

Theorem 3.3. Let $\mathcal{A}$ be an ordered algebra, and let $p$ be a submultiplicative seminorm on $\mathcal{A}$ such that the cone $\mathcal{A}^{+}$is p-normal. Let $a, b$, and $c$ be positive elements in $\mathcal{A}$ satisfying $a c \leq c a, b c \leq c b$, and $a b \leq c b a$. If $c$ is p-power bounded, then

$$
r_{p}(a+b) \leq r_{p}(a)+r_{p}(b) .
$$

Proof. If $\mathcal{A}$ is not unital, then $p_{1}$ is a submultiplicative seminorm on $\mathcal{A}_{1}$ and the cone $\mathcal{A}_{1}^{+}$is $p_{1}$-normal. We also have $a_{1} c_{1} \leq c_{1} a_{1}, b_{1} c_{1} \leq c_{1} b_{1}$, and $a_{1} b_{1} \leq c_{1} b_{1} a_{1}$. Since we also have $r_{p_{1}}\left(x_{1}\right)=r_{p}(x)$ for all $x \in \mathcal{A}$ and $c_{1}$ is $p_{1}$-power bounded, we may assume without loss of generality that $\mathcal{A}$ is unital.

For $n \in \mathbb{N}$ and $k \in \mathbb{N}_{0}$ we define the elements $q_{n, k}(c) \in \mathcal{A}$ in the following way: $q_{n, 0}(c)=q_{n, n}(c)=1$ (the unit element of $\mathcal{A}$ ), and for $k \in \mathbb{N}$ let

$$
q_{n+1, k}(c)=q_{n, k-1}(c)+q_{n, k}(c) c^{k} .
$$

Note that $\left(\begin{array}{l}n \\ k\end{array}\right)+\left(\begin{array}{c}n \\ k-1\end{array}\right)=\left(\begin{array}{c}n+1 \\ k\end{array}\right)$ implies that $q_{n, k}(c)$ is the sum of $\left(\begin{array}{l}n \\ k\end{array}\right)$ (not necessarily distinct) powers of the element $c$.

We will prove by induction on $n$ that

$$
(a+b)^{n} \leq \sum_{k=0}^{n} q_{n, k}(c) b^{n-k} a^{k}
$$


for all $n \in \mathbb{N}$. For $n=1$ the claim is trivially true. Suppose now that inequality (3.7) holds for some $n \in \mathbb{N}$. Then we have

$$
\begin{aligned}
(a+b)^{n+1} & \leq\left(\sum_{k=0}^{n} q_{n, k}(c) b^{n-k} a^{k}\right)(a+b) \\
& \leq \sum_{k=0}^{n} q_{n, k}(c) b^{n-k} a^{k+1}+\sum_{k=0}^{n} q_{n, k}(c) c^{k} b^{n-k+1} a^{k} \\
& =\sum_{k=1}^{n+1} q_{n, k-1}(c) b^{n-k+1} a^{k}+\sum_{k=0}^{n} q_{n, k}(c) c^{k} b^{n-k+1} a^{k} \\
& =\sum_{k=0}^{n+1} q_{n+1, k}(c) b^{n+1-k} a^{k}
\end{aligned}
$$

which completes the induction step.

Inequality (3.7) and the fact that the cone $\mathcal{A}_{+}$is $p$-normal imply that there exists $\gamma>0$ such that

$$
p\left((a+b)^{n}\right) \leq \gamma p\left(\sum_{k=0}^{n} q_{n, k}(c) b^{n-k} a^{k}\right)
$$

for all $n \in \mathbb{N}$. Since $c$ is $p$-power bounded, there exists $M \geq 1$ such that

$$
p\left((a+b)^{n}\right) \leq \gamma M\left(p\left(b^{n}\right)+\sum_{k=1}^{n-1}\left(\begin{array}{l}
n \\
k
\end{array}\right) p\left(a^{k}\right) p\left(b^{n-k}\right)+p\left(a^{n}\right)\right)
$$

for all $n \in \mathbb{N}$. Let us choose now an arbitrary $\epsilon>0$. Then there exists $L>0$ such that for any $n \in \mathbb{N}$ we have $p\left(a^{n}\right) \leq L\left(r_{p}(a)+\epsilon\right)^{n}$ and $p\left(b^{n}\right) \leq L\left(r_{p}(b)+\epsilon\right)^{n}$. Hence, applying (3.8) it follows that

$$
\begin{aligned}
p\left((a+b)^{n}\right) & \leq \gamma M L \sum_{k=0}^{n}\left(\begin{array}{l}
n \\
k
\end{array}\right)\left(r_{p}(a)+\epsilon\right)^{k}\left(r_{p}(b)+\epsilon\right)^{n-k} \\
& =\gamma M L\left(r_{p}(a)+r_{p}(b)+2 \epsilon\right)^{n} .
\end{aligned}
$$

By taking the $n$th roots and letting $n$ go to infinity, we obtain $r_{p}(a+b) \leq r_{p}(a)+$ $r_{p}(b)+2 \epsilon$. Since $\epsilon>0$ was arbitrary, we finally have $r_{p}(a+b) \leq r_{p}(a)+r_{p}(b)$.

The following result follows from Theorems 3.2 and 3.3.

Corollary 3.4. Let $\mathcal{A}$ be an ordered algebra, and let $p$ be a submultiplicative seminorm on $\mathcal{A}$. Let $\mathcal{B}$ be a subalgebra in $\mathcal{A}$ ordered by a p-normal cone $\mathcal{B}^{+}=$ $\mathcal{A}^{+} \cap \mathcal{B}$ which satisfies $\mathcal{A}^{+} \mathcal{B}^{+} \mathcal{A}^{+} \subseteq \mathcal{B}^{+}$. Let $a, b \in \mathcal{A}^{+}$be such that $a b \geq$ ba or $b a \geq a b$. If at least one of the elements $a$ and $b$ is contained in $\mathcal{B}^{+}$, then

$$
r_{p}(a b-b a) \leq r_{p}(a b) \leq r_{p}(a) r_{p}(b)
$$

If $a$ and $b$ are both in $\mathcal{B}^{+}$, then (3.6) holds. 
Proof. As in the proof of Theorem 3.3, we may assume without loss of generality that $\mathcal{A}$ is unital. In this case the result follows from Theorems 3.2 and 3.3 by taking $c$ to be the unit element of $\mathcal{A}$.

Remark 3.5. In particular, Theorem 3.2 and Corollary 3.4 hold whenever $\mathcal{A}$ is an ordered algebra and $p$ is a submultiplicative seminorm on $\mathcal{A}$ such that the positive cone $\mathcal{A}^{+}$is $p$-normal.

\section{Related RESUlts in BANACH ALGEBRAS}

Recall that the spectrum of an element $a$ of a nonunital Banach algebra $\mathcal{A}$ is defined as the spectrum of the element $a_{1}$ in the standard unitization $\mathcal{A}_{1}$ of $\mathcal{A}$. Let $r(a)$ denote the spectral radius of $a \in \mathcal{A}$.

The following theorem is a version of our results in the setting of Banach algebras. Since its proof is very similar to the proofs of Theorem 3.2 and Theorem 3.3, we omit it.

Theorem 4.1. Let $\mathcal{A}$ be a Banach algebra, and let $k \in \mathbb{N}_{0}$. Suppose that the elements $a, b$, and $c$ satisfy $a c=c a, b c=c b$, and $a b=c b a$. If $c$ is power-bounded, then

$$
r(a b) \leq r(a) r(b), \quad r\left(c^{k} b a\right) \leq r(a) r(b), \quad \text { and } \quad r(a+b) \leq r(a)+r(b) .
$$

If $\|c\|<1$, then $a b, b a$, and $c^{k} b a$ are quasinilpotent.

Note that the conditions $a c=c a, b c=c b$, and $a b=c b a$, where $c$ is powerbounded, are natural. Operators satisfying closely related conditions have already been studied in the context of spectral algebras and related invariant subspace problems (see, e.g., [13, Proposition 2.3], [12], [15], [8], and the references therein).

An important example of a Banach algebra is the Calkin algebra. Let $X$ be a Banach space, and let $\pi$ be the canonical projection of $\mathcal{L}(X)$ to the Calkin algebra $\mathcal{L}(X) / \mathcal{K}(X)$, where $\mathcal{L}(X)$ and $\mathcal{K}(X)$ denote the set of all bounded and compact linear operators on $X$, respectively. The essential spectral radius $r_{\mathrm{ess}}(T)$ of the operator $T$ is defined as the spectral radius of the element $\pi(T)$ in the Calkin algebra. An operator $T$ is said to be a Riesz operator whenever $r_{\text {ess }}(T)=0$.

Applying Theorem 4.1 to the Calkin algebra $\mathcal{L}(X) / \mathcal{K}(X)$, we obtain the following result for the essential spectral radius $r_{\text {ess }}$.

Corollary 4.2. Let $X$ be a Banach space, let $k \in \mathbb{N}_{0}$, and let $A, B, C$ be bounded operators on $X$ that satisfy $A C=C A, B C=C B$, and $A B=C B A$. If $C$ is power-bounded, then the following inequalities hold:

$$
\begin{aligned}
& r_{\mathrm{ess}}(A B) \leq r_{\mathrm{ess}}(A) r_{\mathrm{ess}}(B), \quad r_{\mathrm{ess}}\left(C^{k} B A\right) \leq r_{\mathrm{ess}}(A) r_{\mathrm{ess}}(B), \quad \text { and } \\
& r_{\mathrm{ess}}(A+B) \leq r_{\mathrm{ess}}(A)+r_{\mathrm{ess}}(B) \text {. } \\
& \text { If }\|C\|<1 \text {, then } A B, B A \text {, and } C^{k} B A \text { are Riesz operators. }
\end{aligned}
$$




\section{Spectral RADiUs INEQUALITIES FOR POSITIVE OPERATORS}

A linear operator $T$ on an ordered Banach space $E$ is said to be positive whenever the positive cone $E^{+}$is invariant under $T$. An operator $T$ on $E$ is said to be a regular operator (notation $T \in \mathcal{L}_{r}(E)$ ) whenever it is a linear combination of positive operators. A positive operator on an ordered Banach space with a closed and generating cone is bounded (see [4]). In particular, a positive operator on a Banach lattice is bounded. If the cone $E^{+}$of an ordered Banach space is generating, then we can introduce a partial ordering on $\mathcal{L}(E)$ by $A \geq B$ whenever $A-B$ is a positive operator on $E$, so that, in this case, $\mathcal{L}(E)$ with a positive cone $\mathcal{L}^{+}(E)$ becomes an ordered algebra.

Let $E$ be a Banach lattice. The set $\mathcal{L}(E)$ of all bounded operators on $E$ equipped with the operator norm is an example of an ordered normed algebra. It is well known that the operator norm on the positive cone of positive operators on a Banach lattice is monotone.

An operator $T$ on a Banach lattice is said to be an AM-compact operator whenever $T$ maps order intervals to relatively compact sets. It is obvious that every compact operator is an AM-compact operator; however, there exist AM-compact operators that are not compact. For example, the identity operator on $l^{p}$-space $(1 \leq p<\infty)$ is an AM-compact operator that is not a compact operator (see [2, Problem 10.3.7]).

The following theorem states that the cone of positive operators in $\mathcal{L}_{r}(E)$ is normal whenever the cone of the underlying ordered Banach space $E$ is normal, closed, and generating. This result follows immediately from a result of Wickstead [20, Theorem 3.1]. Since it enables us to construct new examples of ordered Banach spaces with normal cones from given ones, we include its short proof.

Theorem 5.1. Let $E$ be an ordered Banach space with a closed, generating, and normal cone. Then the cone of all positive operators on $E$ is a normal cone of $\mathcal{L}_{r}(E)$.

Proof. By the Krein-Šmulian theorem (see [4, Theorem 2.37]), there exists a constant $\rho>0$ such that, for each $x \in E$, there exist positive vectors $y, z \in E$ satisfying

$$
x=y-z, \quad\|y\| \leq \rho\|x\|, \quad \text { and } \quad\|z\| \leq \rho\|x\| .
$$

Since the cone of $E$ is normal as well, [4, Theorem 2.38] implies that there exists a constant $c>0$ such that $\|x\| \leq c \max \{\|y\|,\|z\|\}$ whenever $y \leq x \leq z$.

Let us choose a positive vector $x$ with $\|x\|=1$, and let $A \leq B$ be positive operators on $E$. There exist positive vectors $y, z \in E$ satisfying $x=y-z,\|y\| \leq \rho$, and $\|z\| \leq \rho$. Since the operator $A$ is positive, we have $-A z \leq A y-A z \leq A y$, so that

$$
\begin{aligned}
\|A x\| & =\|A y-A z\| \leq c \max \{\|A y\|,\|A z\|\} \leq c^{2} \max \{\|B y\|,\|B z\|\} \\
& \leq c^{2}\|B\| \max \{\|y\|,\|z\|\} \leq c^{2} \rho\|B\| .
\end{aligned}
$$

Since $x$ with $\|x\|=1$ was arbitrary, we conclude that $\|A\| \leq c^{2} \rho\|B\|$ and that the cone of positive operators is normal in $\mathcal{L}_{r}(E)$. 
Therefore, if positive operators $A, B$, and $C$ on an ordered Banach space $E$ with a closed, normal, and generating cone $E^{+}$satisfy the assumptions of Theorem 3.2, then inequalities $r(A B) \leq r(A) r(B)$ and $r(A+B) \leq r(A)+r(B)$ for the spectral radius hold. In particular, this holds when $E$ is a Banach lattice.

If we omit the assumption that the cone of $E^{+}$is closed, then the cone of positive operators of the space $\mathcal{L}_{r}(E)$ is not necessarily normal. However, inequalities from Theorem 3.2 and Theorem 3.3 still hold (see Theorem 5.2 below). Before we prove this result, we recall some general results on local spectral radii of bounded operators.

Given a real or complex Banach space $X$, the local spectral radius $r_{T}(x)$ of a bounded linear operator $T$ on $X$ at vector $x$ is defined as

$$
r_{T}(x)=\limsup _{n \rightarrow \infty}\left\|T^{n} x\right\|^{\frac{1}{n}} .
$$

It is easy to see that $r_{T}(x) \leq r(T)$ for every $x \in X$. Daneš [6] proved that there exists $x \in X$ with $r_{T}(x)$ equal to $r(T)$. Moreover, he actually proved that the set

$$
\left\{x \in X: r_{T}(x)=r(T)\right\}
$$

is a second category set (by Baire). Förster and Nagy [10] proved that if $E$ is an ordered Banach space with a generating cone $E^{+}$and $T$ is a positive operator on $E$, then

$$
r(T)=\max _{x \in E^{+}} r_{T}(x) .
$$

We present only an outline of the proof of the following result, since we apply similar arguments as in the proofs of Theorems 3.2 and 3.3.

Theorem 5.2. Let $E$ be an ordered Banach space with a normal and generating cone, and let $k \in \mathbb{N}_{0}$. Let $A$ and $B$ be positive operators on $E$, and suppose that there exists a positive power-bounded operator $C$ on $E$ satisfying $A C \leq C A$, $B C \leq C B$, and $A B \leq C B A$. Then

$$
\begin{aligned}
& r\left(C^{k} B A\right) \leq r(A) r(B), \quad r(A B) \leq r(A) r(B), \\
& r(A+B) \leq r(A)+r(B) \quad \text { and } \quad r(C B A-A B) \leq r(C B A) \leq r(A) r(B) .
\end{aligned}
$$

If, in addition, $\|C\|<1$, then the operators $A B, B A, C^{k} B A$, and $C B A-A B$ are quasinilpotent.

Proof. By applying (3.3), the power-boundedness of $C$, and the normality of the cone $E^{+}$, it follows that

$$
r_{C^{k} B A}(x) \leq r(A) r(B)
$$

holds for all $x \in E^{+}$. The inequality $r\left(C^{k} B A\right) \leq r(A) r(B)$ follows from (5.3) and (5.2).

By applying (3.7), the inequality $r(A+B) \leq r(A)+r(B)$ is proved in a similar manner. The rest of the proof is omitted.

Corollary 5.3. Let $E$ be an ordered Banach space with a normal and generating cone. Let $A$ and $B$ be positive operators on $E$ with a positive or negative commutator $A B-B A$. Then we have

$$
r(A B-B A) \leq r(A B) \leq r(A) r(B) \quad \text { and } \quad r(A+B) \leq r(A)+r(B) .
$$


Inequalities $r(A B-B A) \leq r(A B) \leq r(A) r(B)$ appeared in [7] in the case of positive operators $A$ and $B$ with a positive commutator $A B-B A$ on Banach lattices. Zima ([23, Theorem 2.1] and [22]) proved that the same inequalities hold in the case of ordered Banach spaces with normal and generating cones. Moreover, Zima [23] proved that

$$
r(A B+B A)=2 r(A B) .
$$

The following example shows that the assumption on the power-boundedness of the operator $C$ in Theorem 5.2 cannot be omitted.

Example 5.4. Let $E=\mathbb{C}^{2}$,

$$
A=\left[\begin{array}{ll}
0 & 1 \\
1 & 0
\end{array}\right], \quad B=\left[\begin{array}{ll}
0 & 1 \\
4 & 0
\end{array}\right], \quad \text { and } \quad C=4 I .
$$

Then $r(A)=1, r(B)=2$,

$$
C B A=\left[\begin{array}{cc}
4 & 0 \\
0 & 16
\end{array}\right], \quad A B=\left[\begin{array}{ll}
4 & 0 \\
0 & 1
\end{array}\right], \quad A B-B A=\left[\begin{array}{cc}
3 & 0 \\
0 & -3
\end{array}\right]
$$

and

$$
A+B=\left[\begin{array}{ll}
0 & 2 \\
5 & 0
\end{array}\right]
$$

Thus $C B A \geq A B, C A=A C$, and $C B=B C$, but $r(C B A)=16>r(C B A-$ $A B)=15>r(A B)=4>2=r(A) r(B)$ and $r(A+B)=\sqrt{10}>3=r(A)+r(B)$.

Example 5.4 shows also that in Corollary 5.3 the positivity of the commutator cannot be omitted. The following example illustrates that the assumptions of Theorem 5.2 are sensible if we compare them with the assumptions of Corollary 5.3.

Example 5.5. Let $E=\mathbb{C}^{2}$,

$$
A=\left[\begin{array}{ll}
1 & 2 \\
3 & 4
\end{array}\right], \quad B=\left[\begin{array}{ll}
1 & 1 \\
1 & 1
\end{array}\right], \quad \text { and } \quad C=2 I .
$$

Then $r(B)=2, r(A)=0.5(5+\sqrt{33})$,

$$
C B A=\left[\begin{array}{ll}
8 & 12 \\
8 & 12
\end{array}\right], \quad A B=\left[\begin{array}{ll}
3 & 3 \\
7 & 7
\end{array}\right], \quad A B-B A=\left[\begin{array}{cc}
-1 & -3 \\
3 & 1
\end{array}\right]
$$

and

$$
A+B=\left[\begin{array}{ll}
2 & 3 \\
4 & 5
\end{array}\right]
$$

Therefore $A B-B A$ and $B A-A B$ are not positive; $C B A \geq A B, C A=A C$, $C B=B C$, and $r(A B)=10<r(A) r(B) ;$ and $r(A+B)=0.5(7+\sqrt{57})<$ $r(A)+r(B)$. 


\section{Essential SPECTRAL RAdiUs INEQUALITIES FOR POSITIVE OPERATORS}

In this section we apply our general results of Section 3 to the case of the essential spectral radius of positive AM-compact operators on Banach lattices.

The essential spectral radius is closely related to the measure of noncompactness $\beta: \mathcal{L}(X) \rightarrow[0, \infty)$ defined by

$$
\beta(T)=\inf \left\{\delta>0: \exists x_{1}, \ldots, x_{n} \in X \text { such that } T\left(B_{X}\right) \subseteq \bigcup_{i=1}^{n} B\left(x_{i}, \delta\right)\right\} .
$$

Here $B_{X}$ denotes the closed unit ball of $X$, and $B\left(x_{i}, \delta\right)$ denotes the closed ball with the center $x_{i}$ and radius $\delta$.

The measure of noncompactness is a submultiplicative seminorm on $\mathcal{L}(X)$ that satisfies $\beta(T) \leq\|T\|$ (see [1, Lemma 7.59] and its preceding remark; see also [19] or [14]). Nussbaum [16] proved that

$$
r_{\mathrm{ess}}(T)=\lim _{n \rightarrow \infty} \beta\left(T^{n}\right)^{\frac{1}{n}},
$$

which is precisely the $\beta$-spectral radius $r_{\beta}(T)$ of the operator $T$. The proof of the following lemma is straightforward, and so we omit it.

Lemma 6.1. Let $S, T_{1}, T_{2}$, and $R$ be bounded operators on a Banach lattice $E$. If $T_{1}$ and $T_{2}$ are $A M$-compact operators and $R$ is order-bounded, then $S T_{1} R$ and $T_{1}+T_{2}$ are AM-compact operators.

Theorem 6.2. Let $k \in \mathbb{N}_{0}$, let $E$ be a Banach lattice such that $E$ and $E^{*}$ have order-continuous norms, and let $A, B$, and $C$ be positive operators on $E$ satisfying $A C \leq C A, B C \leq C B$, and $A B \leq C B A$. Suppose that the operator $C$ is $\beta$-power-bounded. If at least one of the operators $A$ and $B$ is AM-compact, then

$$
\begin{aligned}
r_{\mathrm{ess}}\left(C^{k} B A\right) & \leq r_{\mathrm{ess}}(A) r_{\mathrm{ess}}(B), \\
r_{\mathrm{ess}}(C B A-A B) & \leq r_{\mathrm{ess}}(C B A) \leq r_{\mathrm{ess}}(A) r_{\mathrm{ess}}(B) .
\end{aligned}
$$

If $A$ and $B$ are $A M$-compact, then

$$
r_{\mathrm{ess}}(A+B) \leq r_{\mathrm{ess}}(A)+r_{\mathrm{ess}}(B) .
$$

If $\beta(C)<1$, then $A B, C^{k} B A$, and $C B A-A B$ are Riesz operators. In particular, if $\|C\|<1$, then $A B, C^{k} B A$, and $C B A-A B$ are Riesz operators.

Proof. Let $\mathcal{B}^{+}$be the set of all positive AM-compact operators on $E$, and let us define $\mathcal{B}:=\mathcal{B}^{+}-\mathcal{B}^{+}$. By Lemma 6.1 the set $\mathcal{B}$ is an ordered algebra and its positive cone $\mathcal{B}^{+}$satisfies $\mathcal{L}_{r}(E)^{+} \mathcal{B}^{+} \mathcal{L}_{r}(E)^{+} \subseteq \mathcal{B}^{+}$. Since the measure of noncompactness is a submultiplicative seminorm on $\mathcal{B}$ which is also monotone on $\mathcal{B}^{+}$by $[17$, Corollary 2.6]; that is, for AM-compact operators $0 \leq S \leq T$ we have $\beta(S) \leq$ $\beta(T)$, and the result follows by applying (6.1) and Theorems 3.2 and 3.3.

Corollary 6.3. Let $A$ and $B$ be positive operators with a positive commutator $A B-B A$ on a Banach lattice $E$ with the property that $E$ and $E^{*}$ have ordercontinuous norms. If at least one of the operators $A$ and $B$ is $A M$-compact, then

$$
r_{\mathrm{ess}}(A B-B A) \leq r_{\mathrm{ess}}(A B) \leq r_{\mathrm{ess}}(A) r_{\mathrm{ess}}(B) .
$$


If $A$ and $B$ are $A M$-compact, then

$$
r_{\mathrm{ess}}(A+B) \leq r_{\mathrm{ess}}(A)+r_{\mathrm{ess}}(B) .
$$

The following result follows directly from Corollary 6.3.

Corollary 6.4. Let $E$ be a Banach lattice such that $E$ and $E^{*}$ have ordercontinuous norms, and let $A$ and $B$ be positive operators on $E$ with a positive commutator $A B-B A$. Suppose that at least one of the operators $A$ and $B$ is AM-compact. Then the following statements hold:

(a) If at least one of the operators $A$ and $B$ is a Riesz operator, then $A B$ and $A B-B A$ are also Riesz operators.

(b) If $A$ and $B$ are $A M$-compact, then the operator $A+B$ is a Riesz operator if and only if $A$ and $B$ are Riesz operators.

Remark 6.5. In Corollary 4.2, we can, by (6.1) and as in Theorem 6.2, replace the assumptions that $C$ is power-bounded and $\|C\|<1$ with weaker assumptions that $C$ is $\beta$-power-bounded and $\beta(C)<1$. We omit the proof.

We continue this section with a result concerning quasinilpotence of positive commutators of positive operators on Banach lattices. In [5], Bračič et al. considered spectral properties of positive commutators of positive operators on Banach lattices. In [5, Theorem 2.2], the authors proved that a positive commutator of positive compact operators is necessarily quasinilpotent, and they asked whether a positive commutator of positive operators is quasinilpotent when only one of the operators $A$ and $B$ is compact. This problem was independently solved in [9] and [11]. Partial results were previously obtained in [7]. We now recall the main result from [7].

Theorem 6.6. Let $A$ and $B$ be positive operators on a Banach lattice $E$ such that $A+B$ is a Riesz operator. If the commutator $A B-B A$ is a power compact positive operator, then it is a quasinilpotent operator.

Since the preceding result does not follow from results obtained in [9] and [11], it is of independent interest, as a positive commutator of positive operators can also be quasinilpotent under different assumptions than the ones from [9] and [11].

The following result extends the class of positive operators with positive quasinilpotent commutators.

Corollary 6.7. Let $E$ be a Banach lattice such that $E$ and $E^{*}$ have order continuous norms, and let $A$ and $B$ be positive $A M$-compact operators on $E$ with a positive commutator $A B-B A$. If $A$ and $B$ are Riesz operators and $A B-B A$ is power-compact, then $A B-B A$ is quasinilpotent.

Proof. Since $A$ and $B$ are Riesz operators, Corollary 6.4 implies that the operator $A+B$ is also a Riesz operator. An application of Theorem 6.6 shows that $A B-B A$ is quasinilpotent.

We conclude this paper with a result on the essential spectral radius version of the equality (5.5). If one of the operators $A$ and $B$ is a compact operator on a Banach space, then we obviously have $r_{\text {ess }}(A B+B A)=r_{\text {ess }}(A B)=0$. The 
equality $r_{\text {ess }}(A B+B A)=2 r_{\text {ess }}(A B)$ is also obvious in the case of commuting operators $A$ and $B$ on a Banach space.

If $E$ is a Banach lattice and $A$ and $B$ are positive power-compact operators on $E$ with a positive commutator $A B-B A$, then the equality $r_{\text {ess }}(A B+B A)=$ $r_{\text {ess }}(A B)=0$ holds as well. Indeed, [7, Lemma 2.2] implies that the operator $A+B$ is power-compact, so that the operator $(A+B)^{2}$ is power-compact as well. Therefore, the operator $(A+B)^{2 n}$ is a compact operator on $E$ for some positive integer $n$. Inequalities

$$
0 \leq A B \leq A B+B A \leq A^{2}+A B+B A+B^{2}=(A+B)^{2}
$$

and [3, Theorem 5.13] imply that $(A B)^{3 n}$ and $(A B+B A)^{3 n}$ are compact operators, so that $A B$ and $A B+B A$ are power-compact operators. It follows that $r_{\text {ess }}(A B+$ $B A)=r_{\text {ess }}(A B)=0$.

We finish this article with the following two results.

Theorem 6.8. Let $E$ be a Banach lattice such that $E$ and $E^{*}$ have ordercontinuous norms, and let $A$ and $B$ be positive operators on $E$ with a positive commutator $A B-B A$. If at least one of the operators $A$ and $B$ is $A M$-compact, then

$$
r_{\mathrm{ess}}(A B+B A)=2 r_{\mathrm{ess}}(A B) .
$$

Proof. The fact that the commutator $A B-B A$ is a positive operator on $E$ implies that

$$
0 \leq 2 B A \leq A B+B A \leq 2 A B \text {. }
$$

By the assumption, operators $B A, A B$, and $A B+B A$ are AM-compact, so that the monotonicity of the essential spectral radius on the positive cone of the ordered algebra generated by positive AM-compact operators gives us

$$
0 \leq 2 r_{\mathrm{ess}}(B A) \leq r_{\mathrm{ess}}(A B+B A) \leq 2 r_{\mathrm{ess}}(A B) \text {. }
$$

To finish the proof we apply the equality $r_{\text {ess }}(A B)=r_{\text {ess }}(B A)$, which holds for all bounded operators on Banach spaces.

Similarly, one can prove the following generalization of Theorem 6.8

Theorem 6.9. Let $\mathcal{A}$ be an ordered algebra, and let $p$ be a submultiplicative seminorm on $\mathcal{A}$. Let $\mathcal{B}$ be a subalgebra in $\mathcal{A}$ ordered by a p-normal cone $\mathcal{B}^{+}=$ $\mathcal{A}^{+} \cap \mathcal{B}$ which satisfies $\mathcal{A}^{+} \mathcal{B}^{+} \mathcal{A}^{+} \subseteq \mathcal{B}^{+}$. Let $a, b \in \mathcal{A}^{+}$be such that $a b \leq$ ba or $b a \leq a b$. If at least one of the elements $a$ and $b$ is contained in $\mathcal{B}^{+}$, then

$$
r_{p}(a b+b a)=2 r_{p}(a b) .
$$

Acknowledgments. The authors thank the anonymous referees for several useful comments and also for pointing out reference [20].

This work was supported in part by grant P1-0222 of the Slovenian Research Agency. 


\section{REFERENCES}

1. Y. A. Abramovich and C. D. Aliprantis, An Invitation to Operator Theory, Amer. Math. Soc., Providence, 2002. Zbl 1022.47001. MR1921782. DOI 10.1090/gsm/050. 142

2. Y. A. Abramovich and C. D. Aliprantis, Problems in Operator Theory, Amer. Math. Soc., Providence, 2002. Zbl 1022.47002. MR1921783. DOI 10.1090/gsm/051. 139

3. C. D. Aliprantis and O. Burkinshaw, Positive Operators, Springer, Dordrecht, 2006. MR2262133. DOI 10.1007/978-1-4020-5008-4. 135, 144

4. C. D. Aliprantis and R. Tourky, Cones and Duality, Amer. Math. Soc., Providence 2007. Zbl 1127.46002. MR2317344. DOI 10.1090/gsm/084. 135, 139

5. J. Bračič, R. Drnovšek, Y. B. Farforovskaya, E. L. Rabkin, and J. Zemánek, On positive commutators, Positivity 14 (2010), no. 3, 431-439. Zbl 1205.47040. MR2680506. DOI 10.1007/s11117-009-0028-1. 143

6. J. Daneš, On local spectral radius, Časopis. Pěst. Mat. 112 (1987), no. 2, 177-187. Zbl 0645.47002. MR0897643. 140

7. R. Drnovšek and M. Kandić, More on positive commutators, J. Math. Anal. Appl. 373 (2011), no. 2, 580-584. MR2720706. DOI 10.1016/j.jmaa.2010.07.056. 141, 143, 144

8. R. Drnovšek and T. Košir, Irreducible operator semigroups such that $A B$ and $B A$ are proportional, Linear Algebra Appl. 383 (2004), 113-118. Zbl 1058.15017. MR2073897. DOI 10.1016/j.laa.2003.11.012. 134, 138

9. R. Drnovšek, Once more on positive commutators, Studia Math. 211 (2012), no. 3, 241-245. Zbl 1267.47062. MR3002445. DOI 10.4064/sm211-3-5. 143

10. K. H. Förster and B. Nagy, "On the local spectral theory of positive operators" in Special Classes of Linear Operators and Other Topics (Bucharest, 1986), Oper. Theory Adv. Appl. 28, Birkhäuser, Basel, 1988, 71-81. MR0942914. 140

11. N. Gao, On commuting and semi-commuting positive operators, Proc. Amer. Math. Soc. 142 (2014), no. 8, 2733-2745. MR3209328. DOI 10.1090/S0002-9939-2014-12002-8. 143

12. M. Lacruz, Invariant subspaces and Deddens algebras, Expo. Math. 33 (2015), no. 1, 116-120. Zbl 1098.47001. MR3310933. DOI 10.1016/j.exmath.2014.04.004. 134, 138

13. A. Lambert and S. Petrović, Beyond hyperinvariance for compact operators, J. Funct. Anal. 219 (2005), no. 1, 93-108. Zbl 1061.47018. MR2108360. DOI 10.1016/j.jfa.2004.06.001. 134, 138

14. P. Meyer-Nieberg, Banach Lattices, Springer, Berlin, 1991. MR1128093. DOI 10.1007/978-3-642-76724-1. 142

15. R. L. Moore, Contractive Commutants and Invariant Subspaces, Proc. Amer. Math. Soc. 83 (1981), no. 4, 747-750. Zbl 0492.47006. MR0630048. DOI 10.2307/2044246. 134, 138

16. R. D. Nussbaum, The radius of the essential spectrum, Duke Math. J. 37 (1970), no. 3, 473-478. Zbl 0216.41602. MR0264434. 142

17. B. de Pagter and A. Schep, Measures of non-compactness of operators in Banach lattices, J. Funct. Anal., 78 (1988), no. 1, 31-55. Zbl 0651.47023. MR0937631. DOI 10.1016/0022-1236(88)90131-0. 142

18. H. Raubenheimer and S. Mouton (née Rode), Cones in Banach algebras, Indag. Math. (N.S.) 7 (1996), no. 4, 489-502. Zbl 1205.47041. MR1620116. DOI 10.1016/S0019-3577(97)89135-5. 135

19. V. G. Troitsky, Measures on non-compactness of operators on Banach lattices, Positivity 8 (2004), no. 2, 165-178. Zbl 1081.47043. MR2097086. DOI 10.1023/B:POST.0000042833.31340.6b. 142

20. A. W. Wickstead, Spaces of linear operators between partially ordered Banach spaces, Proc. London Math. Soc. (3) 28 (1974), 141-158. Zbl 0272.47026. MR0333828. 139, 144

21. M. Zima, On the local spectral radius in partially ordered Banach spaces, Czechoslovak Math. J. 49 (1999), no. 4, 835-841. Zbl pre06343597. MR1746709. DOI 10.1023/A:1022413403733. 
22. M. Zima, Positive Operators in Banach Spaces and Their Applications, Wydawnictwo Uniwersytetu Rzeszowskiego, Rzeszów, 2005. Zbl pre06409104. MR2493071. 134, 141

23. M. Zima, Spectral radius inequalities for positive commutators, Czechoslovak Math. J. 64 (2014), no. 1, 1-10. Zbl pre06391470. MR3247438. DOI 10.1007/s10587-014-0077-x. 134, 141

${ }^{1}$ University of Ljubljana, Faculty of Mathematics and Physics, Jadranska 19, SI-1000 LuUbluana, Slovenia.

E-mail address: marko.kandic@fmf.uni-lj.si

${ }^{2}$ University of Ljubljana, Faculty of Mechanical Engineering, Aškerčeva 6, SI-1000 LuUbluana, Slovenia.

E-mail address: aljosa.peperko@fs.uni-lj.si; aljosa.peperko@fmf.uni-lj.si 